\title{
PAPR Reduction in OFDM Systems Using Hybrid Zadoff-Chu Transform Precoding and Partial Transmit Sequence
}

\author{
M. A. Oyegoke and Y. O. Olasoji
}

\begin{abstract}
Orthogonal Frequency Division Multiplexing (OFDM) is a multicarrier modulation (MCM) scheme that plays a significant role in digital wireless communication. As a result of its high data rate capability and immunity to multipath fading effect, among others, OFDM remains an ideal technology for 5G communication systems and beyond. Unfortunately, a major drawback of this technology is the high peak-to-average power ratio (PAPR). Nevertheless, many techniques for reducing PAPR have been proposed in the past to address this problem. However, all the techniques previously used have limitations such as high computational complexity, data rate loss, high signal distortion, increase in bandwidth, increase in transmit power, and memory requirements. In this paper, a hybrid technique that combines Zadoff-Chu Transform (ZCT) precoding and Partial Transmit Sequence (PTS) for reducing high PAPR in OFDM signals is presented. To reduce the data rate loss and computational complexity in PTS when a large subblock is used, a Zadoff-Chu precoding is applied to OFDM symbols to precode the symbol before applying PTS with a fewer number of subblocks. An OFDM model was developed where ZCT and PTS techniques were implemented. The performance of the hybrid model was analyzed using Power Spectral Density (PSD), Complementary Cumulative Distribution Function (CCDF), and Bit Error Rate (BER). The simulation result indicates that the hybrid ZCT-PTS provides a better result than using either Zadoff-Chu precoding technique or Partial Transmit Sequence technique separately. A PAPR of $4.2 \mathrm{~dB}$ and BER of $3 \mathrm{~dB}$ is achieved for hybrid ZCT-PTS. The hybrid technique provides a better system performance when compared with Conventional OFDM systems.
\end{abstract}

Keywords - 5G; beyond 5G (B5G); hybrid ZCT-PTS orthogonal frequency division multiplexing (OFDM); partial transmit sequence (PTS); peak to average power ratio (PAPR); Zadoff-Chu precoding.

\section{INTRODUCTION}

With the evolution of wireless communication, there has been an enormous increasing demand for high data rates. This is due to an increase in multimedia services and applications e.g. real-time streaming, multiplayer games, other social media services, High Definition (HD), and tactile internet [1], [2]. OFDM is a major form of digital MCM scheme which is regarded as a better technique for providing high-speed data rate in wire-line and wireless communication systems [3], [4]. It offers high spectral efficiency, high data rate capacity, bandwidth flexibility, robustness to multipath fading, is easy to implement based on inverse Fast Fourier Transform (IFFT) algorithm, and the ability to combine with other systems of the same band [5]-[7]. Furthermore, OFDM is compatible with Multiple-Input-Multiple-Output (MIMO) technologies which are expected to be an important scheme in $5 \mathrm{G}$ systems [8], [9], [10]. However, despite the numerous advantages of OFDM, the major drawback is its high PAPR. This high PAPR is formed by subjecting OFDM symbols to IFFT operation. ${ }^{1}$ During this process, a subset of these symbols adds up coherently. This leads to the creation of large peaks at the output [11]. This high PAPR causes non-linear distortion in high power amplifiers because of its large magnitude spikes making the OFDM system to be undesirable. [4], [12]. As a result of this, it becomes a necessity to reduce the high PAPR of the OFDM signal before transmission. This high PAPR problem has been widely studied in OFDM systems, and different reduction methods have been proposed to reduce it. These techniques involve a tradeoff between computational complexity, level of signal distortion, and spectral efficiency. The tradeoff created by several PAPR reduction techniques becomes another critical issue as it can limit the implementation of OFDM in $5 \mathrm{G}$ or beyond (B5G). In recent years, hybrid PAPR reduction techniques have gained interest and research studies in this domain may be found in [13]. These hybrid techniques employ the advantages present in two or more techniques for better PAPR reduction and better system performance.

In this paper, a new hybrid technique for reducing PAPR is presented. The technique employs ZCT precoding and PTS to achieve a reduction in PAPR of OFDM system. The performance of the hybrid technique was evaluated using BER, CCDF, and PSD.

The rest of this work is organized as follows: Section II presents OFDM system model with mathematical analysis of PAPR. In section III, Zadoff-Chu matrix based PAPR reduction technique is presented, while section IV presents PTS based OFDM system. In section V, the proposed Hybrid PAPR reduction technique is presented. Section VI presents simulation results and discussions while the conclusion is presented in section VII.
Submitted on September 17, 2021

Published on October 12, 2021.

M. A. Oyegoke, Department of Electrical and Electronics Engineering, School of Engineering and Engineering Technology, The Federal University of Technology, Akure, Ondo State, Nigeria.

(corresponding e-mail: oyegoke.ayomipo10@gmail.com).
Y. O. Olasoji, Department of Electrical and Electronics Engineering, School of Engineering and Engineering Technology, The Federal University of Technology, Akure, Ondo State, Nigeria.

(e-mail: yoolasoji@futa.edu.ng). 


\section{OFDM SYSTEM AND PAPR ANALYSIS}

The block diagram of OFDM system is shown in Fig. 1. Modulation or mapping is firstly performed on the successive number of input data symbols by different M-ary modulators (QPSK QAM and BPSK). The $N$ complex data symbol is then connected and transferred by serial to parallel (S/P) converter. Using IFFT, the parallel data is jointly connected at the transmitter end. IFFT produces orthogonal data subcarriers. In (1), the data block of length $N$ is represented by a vector.

$X_{k}=X(0), X(1), \ldots X(N-1)^{T}$

where the duration of any symbol $X_{K}$ in the set, $X$ is $T$ and represents one of the sub-carriers set. Applying IFFT to the block of length $N$ results into $\{\mathrm{x}(0), x(1), \ldots \ldots x(N-1)\}$ which are the samples of transmitted information symbols $X$. As the $N$ sub-carriers chosen, the transmit signal are orthogonal to each other. where $f_{n}=n \Delta f, n \Delta f=\frac{1}{N T}$ and $N T$

is the duration of the OFDM data block $X$. The transmitted complex data of the OFDM signal is given in (2) as presented by Han and Lee [14]

$x(t)=\frac{1}{\sqrt{N}} \sum_{k=0}^{N-1} X_{k} e^{j 2 \pi n \Delta f t} 0 \leq N T$

where $k=(0,1 \ldots, N-1)$ block of $N$ input bits symbol to be transmitted on the $\mathrm{k}^{\text {th }}$ subcarrier, $j=\sqrt{-1}, N$ is the total number of subcarriers $X_{k}, \Delta f$ is the subcarrier spacing, $N T$ is the useful data block period, and $T$ is the transmit symbol time. The subcarriers in OFDM are orthogonal to each other (i.e., $\Delta f=\frac{1}{N T}$ ). The discrete form of OFDM signal $x(n)$ is presented in (3):

$x(n)=\frac{1}{\sqrt{N}} \sum_{n=0}^{N-1} X_{k} e^{\frac{j 2 \pi k n}{N}}$ for $n=0,1,2, N-1$

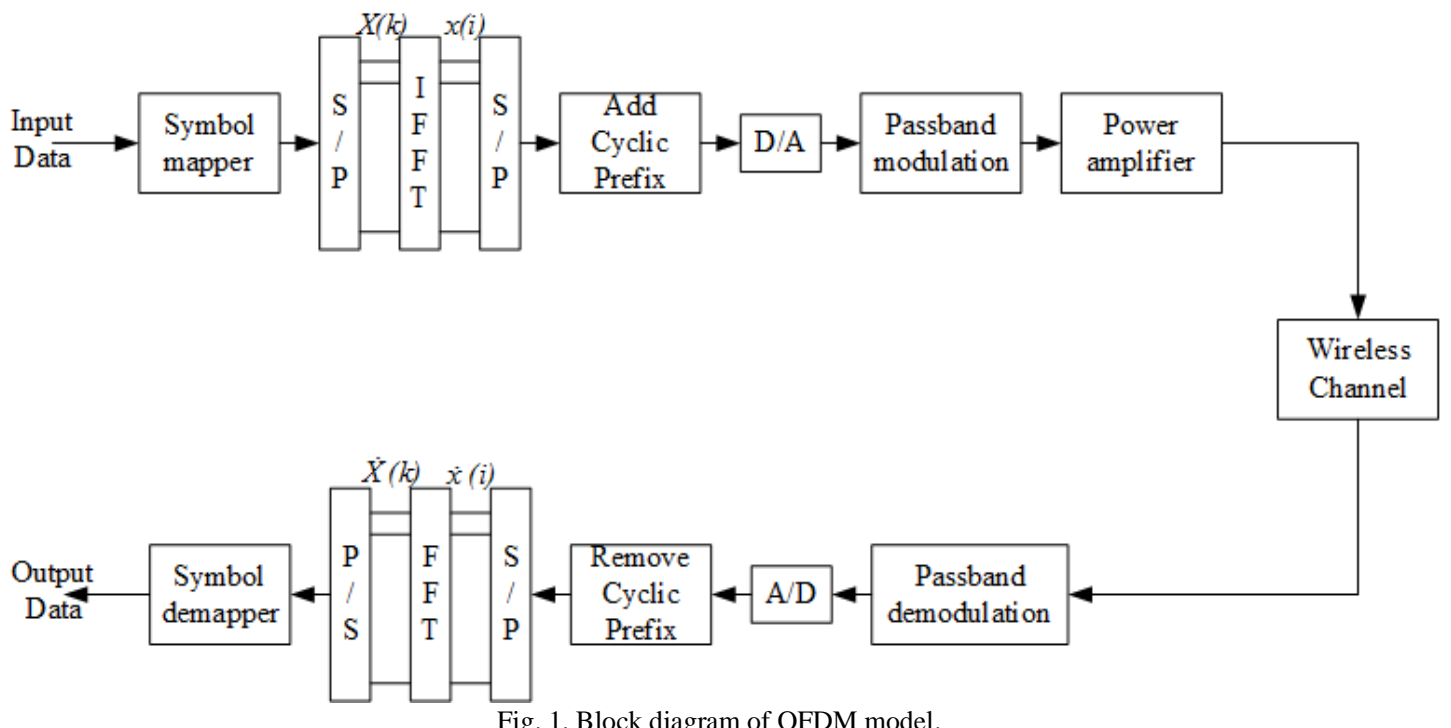

\section{A. Analysis of PAPR}

PAPR is an important factor to be considered in designing a high-power amplifier (HPA) as each HPA is an essential part of wireless communication. Jiang and $\mathrm{Wu}$ [15] mathematically defined PAPR as the ratio of Peak power to Average power. That is:

PAPR $=\frac{\text { Peak Power }}{\text { Average Power }}$

From (2), the OFDM signal is generated using input symbols $X_{k}$, the PAPR of the continuous-time baseband OFDM transmitted signal $x(t)$ is the ratio of the maximum instantaneous power and the average power.

By definition,

$$
P A P R=\frac{\max [x(t)]^{2}}{E\{|x(t)|\}^{2}}, \text { for } 0 \leq t \leq N T
$$

where $E\{$.$\} denotes Expectation operator, N$ is the number of subcarrier and $E\{|x(t)|\}^{2}$ is the average power of $x(t)$ as well as $T$ is an original symbol period. For a discrete OFDM signal $x(n)$, this is represented in (6):
$P A P R=\frac{\max [x(n)]^{2}}{E\{|x(n)|\}^{2}}$, for $0 \leq t \leq N L-1$

where $N$ is the total number of sub-carriers and $E\{|x(n)|\}^{2}$ is the average power of $x(n)$. The corresponding PAPR can be defined as:

$\operatorname{PAPR}(x)=10 \log _{10} \frac{\max [x(n)]^{2}}{E\{|x(n)|\}^{2}}$

\section{B. PAPR distribution}

The Cumulative Distribution Function (CDF) is one of the most regularly used parameters to measure the efficiency of any PAPR technique. Normally, the CCDF is used instead of CDF which helps us to measure the probability that the PAPR of a certain data block exceeds the given threshold. The CDF of the amplitude of a signal sample is given by:

$F(Z)=\left(1-e^{-z^{2}}\right)$

where $Z$ is a magnitude of complex samples. The CCDF of the PAPR for the non-oversampling data block is given as: 
$P(P A P R>Z)=1-F(Z)^{N}=1-\left(1-e^{-z^{2}}\right)^{N}$

where $N$ is assumed to be a large number of sub-carriers. The CCDF of the PAPR for oversampling $(L)$ data block is obtained by multiplying (8) with $N$ and $L$. This can be further expressed as:

$$
C C D F=P(P A P R>z) \approx 1-\left(1-e^{-z^{2}}\right)^{N L}
$$

\section{ZADOFF-CHU MATRIX BASED OFDM SYSTEM}

ZCT OFDM as shown in Fig. 2 is one of the methods of precoding the input data symbol for PAPR reduction in OFDM system. It is important to state what Zadoff-Chu precoding entails before carrying out the performance on the OFDM system.

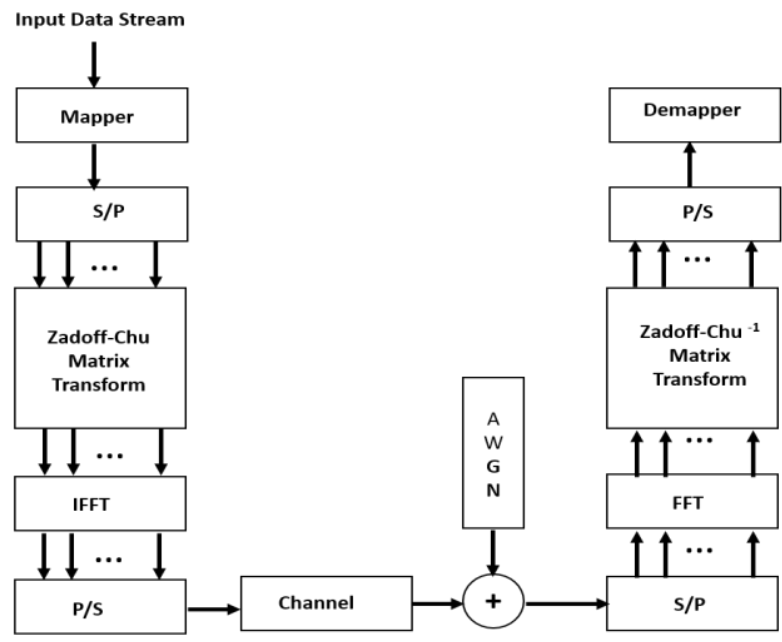

Fig. 2. Block diagram of ZCT precoding based OFDM system.

\section{A. Zadoff-Chu Sequences}

According to Popovic [16], Zadoff-Chu sequences of length $N$ can be represented in (10). Zadoff-Chu sequences are a class of polyphase sequences with optimum correlation properties. Zadoff-Chu sequences have an ideal periodic autocorrelation and constant magnitude.

$p_{n}=\left\{\begin{array}{l}e^{\frac{j 2 \pi r}{N}\left(\frac{k^{2}}{2}+q k\right), \text { for } N \text { even }} \\ e^{\frac{j 2 \pi r}{N}\left(\frac{k(k+1)}{2}+q k\right), \text { for } N \text { odd }}\end{array}\right.$

where $k=0,1,2, \ldots N-1, q$ is any integer, $r$ is an integer relatively prime to $N$ and $j=\sqrt{-1}$.

\section{B. Zadoff-Chu Matrix Transform (ZCT)}

The kernel of the ZCT is defined in (11). The ZCT $P$, of size $N=L \times L$ is obtained by reshaping the ZC sequence with $k=m+l L$ as hereunder:

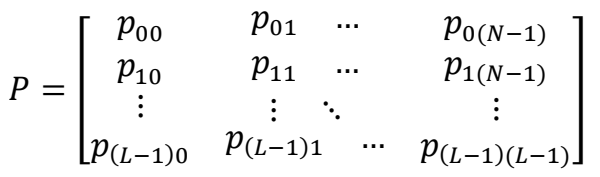

$P$ is the precoder matrix of the Zadoff-Chu sequence. Here, $m$ is the row variable and 1 are the column variable. In other words, the $N=L \times L$ point long ZC sequence fills the kernel of the matrix column-wise. The block diagram of the ZCT precoded OFDM system is depicted in Fig. 2 and the following operations were carried out:

(i) The input data streams generated using MATLAB were modulated by constellation symbol (QPSK, 16QAM, and 64QAM), and the output of the modulation is passed through $\mathrm{S} / \mathrm{P}$ converter to generate a complex vector of size $N$ which is written as:

$X=\left[X_{0}, X_{1}, X_{2}, \ldots, X_{N-1}\right]^{T}$

(ii) ZCT precoding matrix is applied to complex vector to form a new vector of length $N$, the new vector transformed by $\mathrm{ZCT}$ precoding matrix is represented as:

$Z=P X=\left[Z_{0}, Z_{1}, Z_{2} \ldots, Z_{N-1}\right]^{T}$

where $P$ is defined as the precoder matrix of size $N \times N$ and $Z_{m}$ is written as:

$Z_{m}=\sum_{n=0}^{N-1} p_{m, n} X_{n}, m=0,1,2, \ldots, N-1$

where $p_{m, n}$ means $m^{\text {th }}$ row and $n^{\text {th }}$ column of precoder matrix. Expanding (15) by using column-wise reshaping $k=$ $m+n N$ and putting $q=0$ and $r=1$ in (14) gives (15):

$Z_{m}=\sum_{n=0}^{N-1} e^{j \frac{\pi(m+n N)^{2}}{N^{2}}} X_{n}=e^{j \frac{\pi m^{2}}{N^{2}}} \sum_{n=0}^{N-1} X_{n} e^{j \pi n^{2}} e^{j \frac{\pi m n}{N}}$

where $m=0,1,2, m, \ldots, N-1$. The ZCT precoded constellation symbol is represented in (15).

(iii) The complex baseband ZCT-OFDM signal with $\mathrm{N}$ subcarriers when applying with Inverse Fast Fourier transform can be written as:

$x_{n}=\frac{1}{\sqrt{N}} \sum_{m=0}^{N-1} Z_{m} e^{j 2 \pi \frac{n}{N} m} n=0,1,2, \ldots L-1$

\section{Partial Transmit SEQUENCE}

The PTS technique is one of the popular and most efficient techniques of the multiple signaling and probabilistic method. In this technique, the input data sequence is divided into a set of non-over lapping sub-sequences, and IFFT is applied to each of them independently. The outputs are then scaled, and phase rotated by a set of different phase factors, and the combination which yields the least PAPR is selected for transmission [17]. In the PTS, the number of IFFT and subblocks are the same. Better PAPR reduction is achieved in PTS technique when compared with other distortionless techniques such as interleaving technique and selective mapping (SLM) [14]. However, the disadvantages of this technique are that it suffers from computational complexity when a large number of subblocks is chosen and also suffers from data rate loss when side information is transmitted with OFDM data.

In Fig. 3, the block diagram of PTS technique is depicted. The following operations were carried out during the implementation into OFDM system:

(i) The input data block $X$ of $N$ symbols is separated into $V$ disjoint sub-blocks represented as $\left\{X_{v}, v=\right.$ 
$0,1,2,3 \ldots V-1\}$. The input data block $X$ can be written in the form as expressed in (17).

$X=\sum_{v=0}^{V-1} X_{V}$

where $X_{v}=\left\{X_{v}{ }^{0}, X_{v}{ }^{1}, X_{v}{ }^{2} \ldots \ldots \ldots \ldots X_{v}{ }^{N-1}\right\}^{T}$ are the subblocks of the same size.

(ii) The subblocks were partition by pseudo-random partitioning methods, pseudo-random and each subblock have equal size. Each partitioned subblock $X_{v}$ is multiplied by a complex phase factor $b_{v}=e^{j v \theta}, v=$ $1,2,3 \ldots V$. subsequently transforming into $V$ time-domain partial transmit sequence by taking the $L N$ - point IFFT, this is represented in (18):

$x=\operatorname{IFFT}\left(\sum_{v=1}^{V} b_{v} X_{v}\right)=\sum_{v=1}^{V} b_{v} \cdot \operatorname{IFFT}\left(X_{v}\right)=$

$\sum_{p=1}^{P} b_{v} x_{v}$

where $\left(x_{v}\right)$ is a Partial Transmit Sequence (PTS). The phase factor should be chosen in such a way that PAPR can be minimized as given in (19):

$\left.\widehat{b_{0}} \ldots \ldots \ldots \widehat{b_{v-1}}\right]=$

$\arg \min _{b_{0} \ldots \ldots b_{v-1}}\left(\max _{n=0,1, \ldots . . L N-1}\left|\sum_{b=0}^{V-1} b_{v} x_{v}[n]\right|\right)$

where $n=(0,1,2, L N-1)$ and $L$ is the oversampling factor. The corresponding time-domain signal with the lowest PAPR vector can be expressed in (20):

$\hat{x}=\sum_{p=0}^{p-1} \hat{b}_{v} x_{v}$

(iv) The selection of the phase factors $\left(b_{v}\right)_{v=1}^{V}$ is limited to a set of elements to reduce the search complexity.

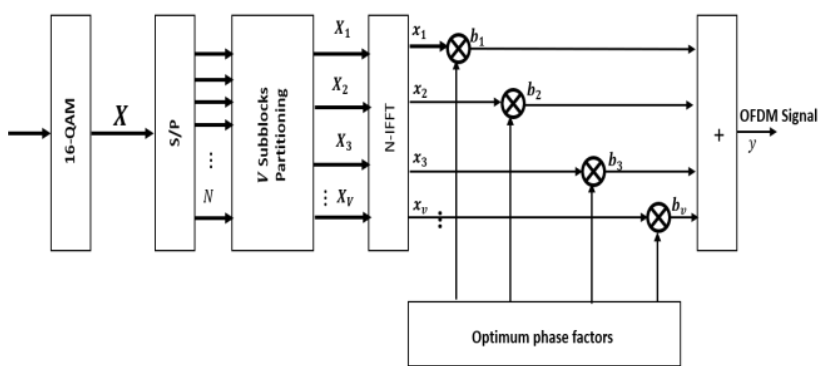

Fig. 3. Block diagram of partial transmit sequence.

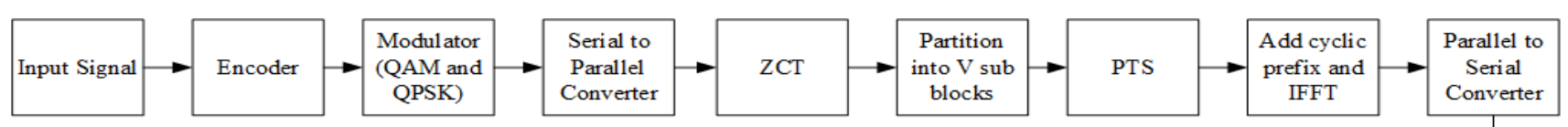

selecting the appropriate phase factor, the lowest PAPR vector in the time domain is given as: $\check{x}=\sum_{m=1}^{V} \check{w}_{v} * x_{v}$

where, $P_{m, n}$ is the element of precoder matrix $P$ as expressed in (21).

(iii)

The

precoded

symbols

$\left[X_{p}(0), X_{p}(1), X_{p}(2) \ldots X_{P}(L-1)\right]^{T}$ are oversampled by a sampling factor $L_{S}$ to provide a better estimation of PAPR by inserting $\left(L_{S}-1\right) N$ zeros in the precoded symbol. These oversampled precoded symbols are partition into $V$ disjoint sub-blocks:

$X_{v}=\sum_{m=1}^{V} X_{p}$

(iv) Pseudorandom partitioning scheme is applied to separate subblocks. Then $V$ subblocks are multiplied by a complex phase factor $b_{v}=\exp (j v \theta)$, where $v=1,2,3 \ldots V$.

(v) The output from the partitioning is multiplied by IFFT of each subblock at the same time.

$x=\sum_{m=1}^{V} b_{v} \times \operatorname{IFFT}\left\{X_{v}\right\}=\sum_{m=1}^{V} b_{v} * x_{v}$

$x_{v}$ is called partial transmit sequence (PTS). After

Zadoff-Chu precoding-based OFDM of encoded data applying to PTS OFDM with $N$ sub-carriers is shown in Fig. 4. The following operation was carried out in hybridizing Zadof-Chu Precoding and Partial Transmit Sequence

(i) M-ary data encoder converts the input data bits into QAM modulation techniques were used for the data encoder. After that, encoded data $(X)$ with data rate $^{1} / T_{S}$ is arranged (ii) These symbols $N$ are converted from serial to parallel. Each $N$ symbols block is precoded by precoding matrix of

$X_{P}=\sum_{n=0}^{N-1} p_{m, n} X_{n}, m=0,1,2, \ldots, N-1$

(n)


(vi) At the receiver, the received signal $Y=$ $[Y(0), Y(1), \cdots Y(L N-1)]^{T}$ is partitioned again into disjoint subblocks using the same partition scheme of the transmitter side. Each partition sub-blocks are multiplied by their optimized phase factors $b^{*}=\left[b_{1}^{*}, b_{2}^{*}, \cdots b_{v}^{*}\right]$ which are calculated on the transmitter side, where $b^{*}$ is a transpose of $b$. Each time-domain subblock is converted to the frequency domain by using $L N$-point FFT. The Frequency domain subblock is multiplied with a matrix $P^{*}$, where $P^{*}$ is the hermitan transpose of $P$. After the multiplication, a vector of length $N$ is obtained:

$\check{X}=P^{*} \times Y$, for $n=0,1,2, \cdots N-1$

Then, the obtained result of vector $\check{X}$ is decoded by using different decoded schemes (M-QAM) to recover transmitted symbols.

\section{Simulation Results AND DisCUSSIONS}

Computer simulations using MATLAB for the evaluation of the proposed hybrid technique is performed in this section. The proposed hybrid technique is compared with ZCT, PTS and original OFDM signal. The OFDM model is simulated using AWGN as a channel with cyclic prefix as a guard band. The input data streams are modulated by QPSK, 16-QAM and 64-QAM with subcarrier $N=16,64,128,256$, and 512 . The performance of proposed hybrid ZCT-PTS PAPR reduction technique is evaluated using $\mathrm{CCDF}$, BER and PSD. The parameters used in simulation are listed in Table I.

\begin{tabular}{cc} 
TABLE I: PARAMETERS USED IN SIMULATION OF OFDM PAPR \\
REDUCTION \\
\hline Parameters used & Values \\
\hline Number of subcarriers $(N)$ & $16,64,128,256,512$ \\
Oversampling factor $(L)$ & 4 \\
Phase rotation factors $(b)$ & $(+1,-1)$ \\
Number of subblocks $(V)$ & $2,4,8$ \\
Modulation scheme & QPSK, 16 QAM, 64 \\
Number of generated OFDM & QAM \\
Symbol & $10^{5}$ \\
Guard type & Cyclic prefix \\
Channel type & AWGN \\
Cyclic prefix length & 16,64 \\
&
\end{tabular}

In Fig. 5, the CCDF of the OFDM PAPR for different numbers of $(N)$ with a 16-QAM modulation scheme is presented. The modulated data is oversampled by a factor of $(L=4)$ with a cyclic prefix (CP) length of 16. It is observed that at $N=16$, the lowest PAPR of $10.34 \mathrm{~dB}$ is obtained. With the increment of $N$, there is an increase in the value of the PAPR and it aligns on the same point on the graph. The highest PAPR of $10.89 \mathrm{~dB}$ is achieved as $N$ increases from 64 to 512 .

The CCDF of PAPR of OFDM for different values of subcarrier $(N)$ using the 64-QAM modulation scheme is presented in Fig. 6. The modulated data is oversampled by a factor of 4 with a CP length of 16 . The result from the graph shows that with the increment in $N$, there is a corresponding increment in the PAPR. With the trend in the graph moving from $N=64$ to 512 , the result shows that the increment is negligible as they all fall on the same point in the graph.

In Fig. 7, the CCDF of the OFDM PAPR for different numbers of subcarrier $(N)$ using QPSK is presented. The modulated data is oversampled by a factor of 4 with a cyclic prefix length of 16 . The trend in the graph shows that at $N=$ 16 , the lowest PAPR of $10.74 \mathrm{~dB}$ is achieved. It is also observed that at $N=64,128$, and $256,10.9 \mathrm{~dB}$ of PAPR is achieved while at $N=512$, the PAPR is $10.85 \mathrm{~dB}$.

Fig. 8 presents the CCDF of the OFDM PAPR for $N=64$ which are oversampled by an oversampling factor of 4 using a 16-QAM modulation scheme. The graph shows that using the ZCT precoding, there is a reduction in the OFDM PAPR from $10.84 \mathrm{~dB}$ to $4.8 \mathrm{~dB}$. This amount to about $56 \%$ reduction. The result shows that the $\mathrm{ZCT}$ precoding technique is efficient to reduce OFDM PAPR as almost $6 \mathrm{~dB}$ is reduced.

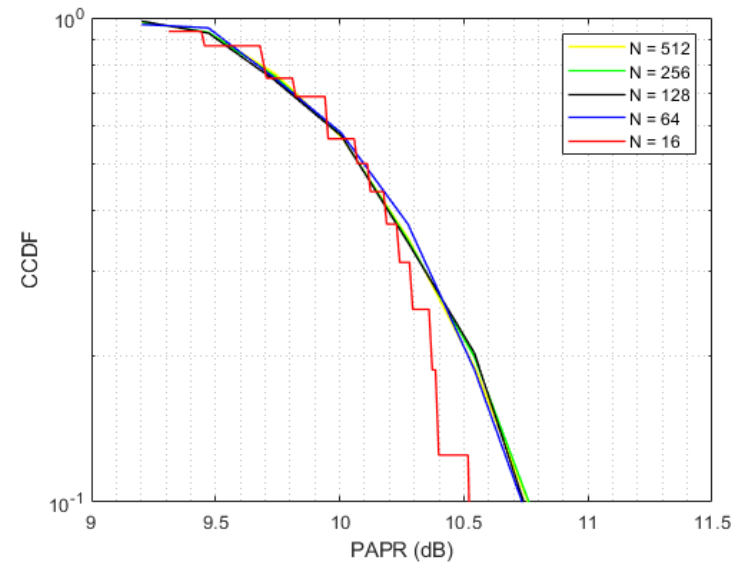

Fig. 5. CCDF of the OFDM PAPR for different $N$ with 16-QAM.

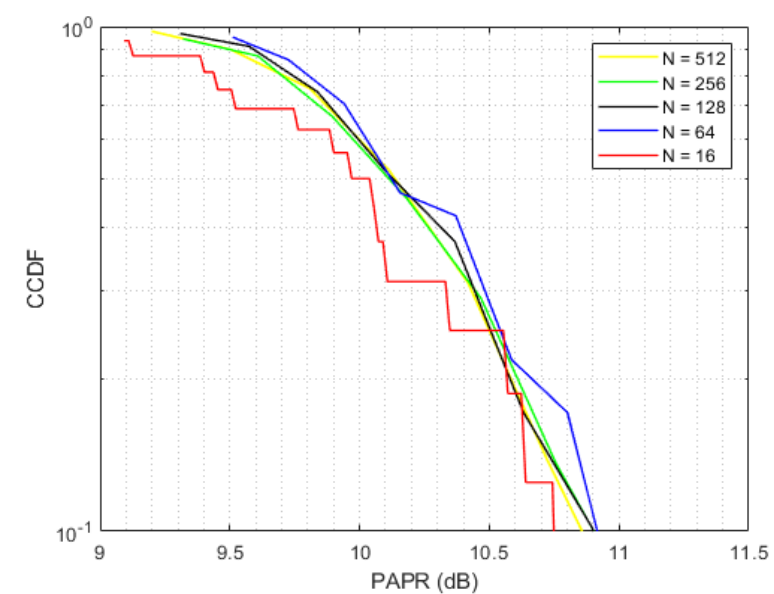

Fig. 6. CCDF of the OFDM PAPR systems for different $N$ with 64-QAM.

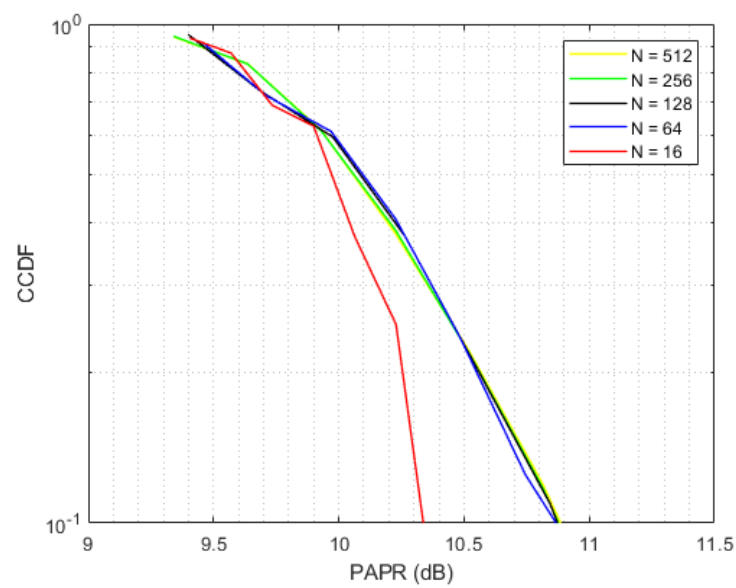

Fig. 7. CCDF of the OFDM PAPR for different $N$ with QPSK 


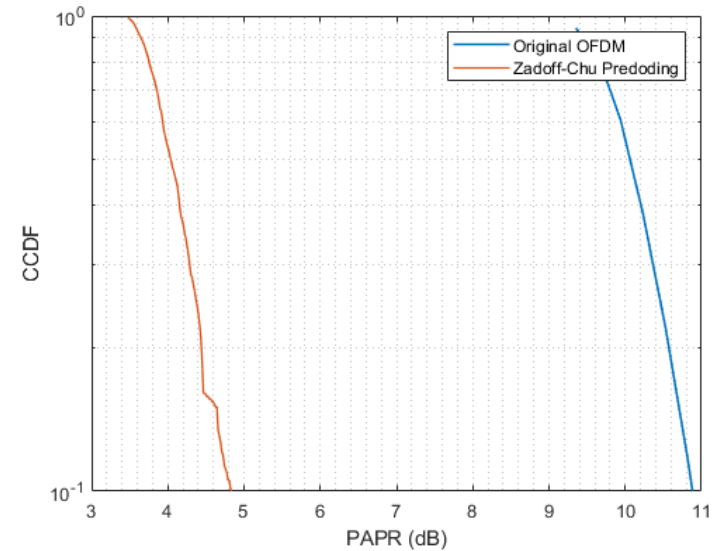

Fig. 8. CCDF of conventional OFDM and Zadoff-Chu precoding method $(N=64$ and $L=4)$.

In Fig. 9, the result of CCDF of the PAPR of the developed hybrid ZCT-PTS for $N=64$ subcarriers by an oversampling factor of 4 using a 16-QAM modulation scheme is presented. The graph shows the efficiency of the developed hybrid ZCTPTS. There is a reduction in the PAPR of the original OFDM signal from $10.84 \mathrm{~dB}$ to $4.2 \mathrm{~dB}$. The result shows that the developed hybrid ZCT-PTS is better for PAPR reduction in OFDM system as $6.64 \mathrm{~dB}$ is reduced.

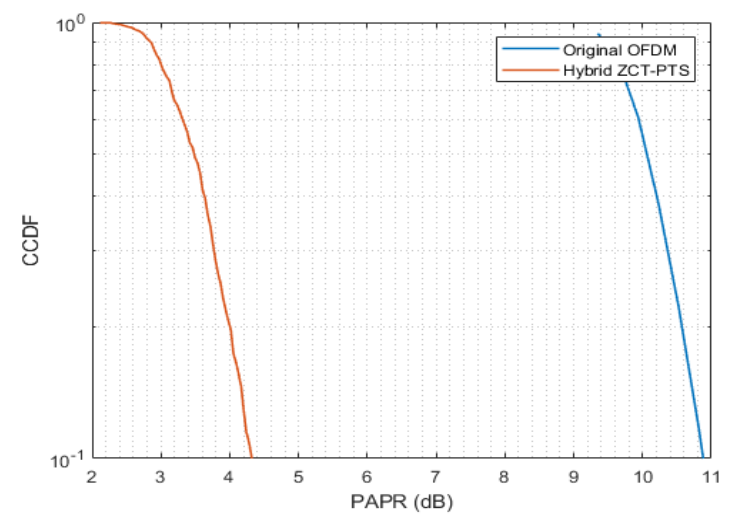

Fig. 9. CCDF of hybrid ZCT-PTS and conventional OFDM using 16-QAM.

Fig. 10 shows the CCDF of the PTS technique at a different number of subblocks $(V=2,4,8)$. Pseudo random segmentation is used as the partitioning scheme. The most obvious trend in the graph is that PAPR goes on decreasing as the number of subblocks increases. 16-QAM modulation scheme was used at $N=64$. The least PAPR of $4.4 \mathrm{~dB}$ is obtained at $C C D F=10^{-1}$ when $V=8$. The PTS method with subblocks $V=2$ and 4 has a peak value of approximately $7.85 \mathrm{~dB}$ and $5.82 \mathrm{~dB}$ respectively. The result obtained from the graph shows that with the increment in $V$, the PAPR decreases but the systems' computational complexity increases. For better system performance, the choice of $V$ must be carefully selected.

Fig. 11 presents the comparison of BER of hybrid ZCTPTS technique with original OFDM signal. It can be seen from the graph that original OFDM and hybrid ZCT-PTS technique provide $3 \mathrm{~dB}$ and $18 \mathrm{~dB}$ respectively. In comparison with the original OFDM signal, the developed hybrid ZCT-PTS technique offers less of SNR (dB).

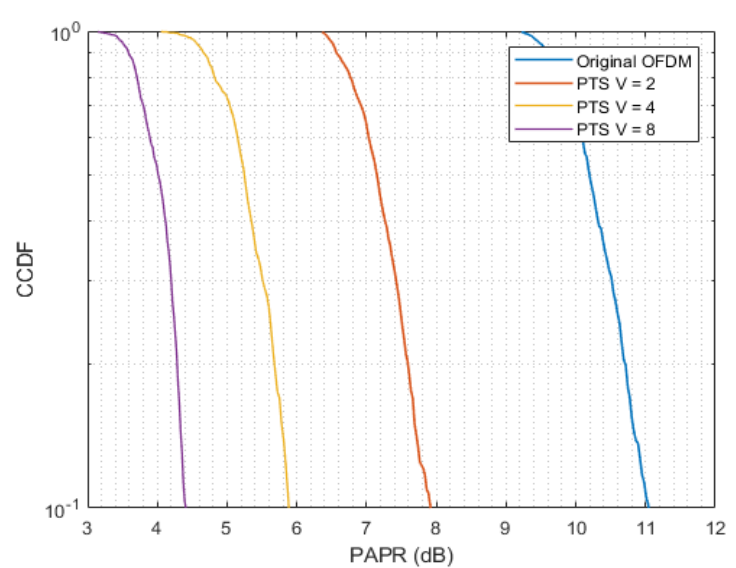

Fig. 10. CCDF of conventional OFDM and PTS technique showing different subblocks $(V=2,4,8)$ when $N=64$.

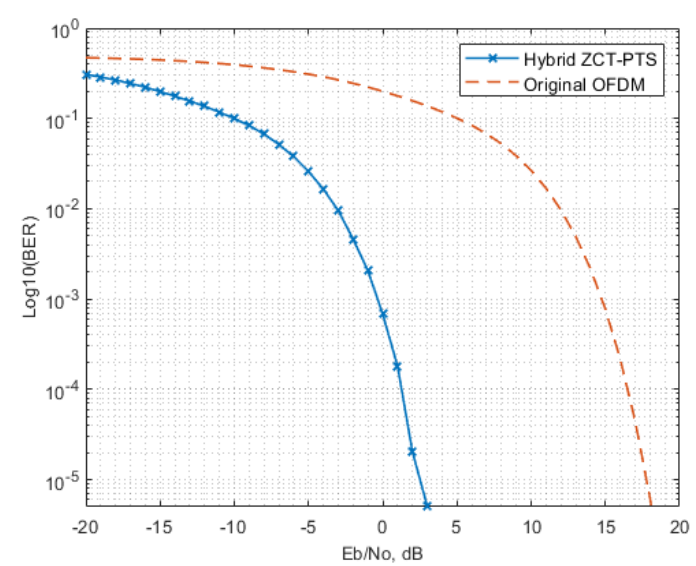

Fig. 11. BER of conventional OFDM and hybrid ZCT-PTS

In Fig. 12, the CCDF of a conventional OFDM signal, the Zadoff-Chu precoding technique, the PTS technique at different subblock levels ( $V=2,4$, and 8), and the developed hybrid technique is presented. Simulation is done using 16QAM modulation and partitioning of Subblock is done by Pseudorandom partitioning. The developed hybrid model is simulated by combining Zadoff-Chu precoding and PTS when subblock $V=4$. The trend in the graph shows that for the PTS technique, as the subblocks $(V)$ increases, the PAPR decreases but the computational complexity of PTS scheme increases. It can be seen from the graph that the original OFDM PAPR is $10.84 \mathrm{~dB}$, developed hybrid ZCT-PTS has the lowest PAPR of $4.2 \mathrm{~dB}$, Zadoff-Chu precoding with 4.8 $\mathrm{dB}$, and PTS with different subblocks of $2,4,8$ has PAPR of $7.85 \mathrm{~dB}, 5.82 \mathrm{~dB}, 4.4 \mathrm{~dB}$, respectively.

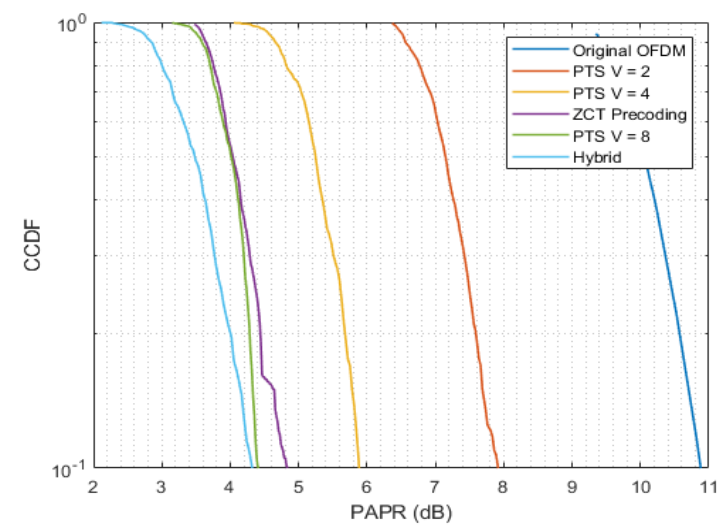

Fig. 12. CCDF of conventional OFDM, hybrid ZCT-PTS, PTS $(V=2,4,8)$ and ZCT precoding. 
In Fig. 13, the PSD of original OFDM and hybrid ZCTPTS is presented. As can be noticed in this figure, the Power spectral density of the developed hybrid ZCT-PTS is approximately $-58 \mathrm{~dB} / \mathrm{MHz}$ at its highest peak within the range of -2 to $2 \mathrm{MHz}$ while the conventional OFDM is approximately $-53 \mathrm{~dB} / \mathrm{MHz}$ at its highest peak within the range of 2 to $-2 \mathrm{MHz}$. From the observation, it can be seen that the developed hybrid ZCT-PTS performs better when compared with the conventional OFDM.

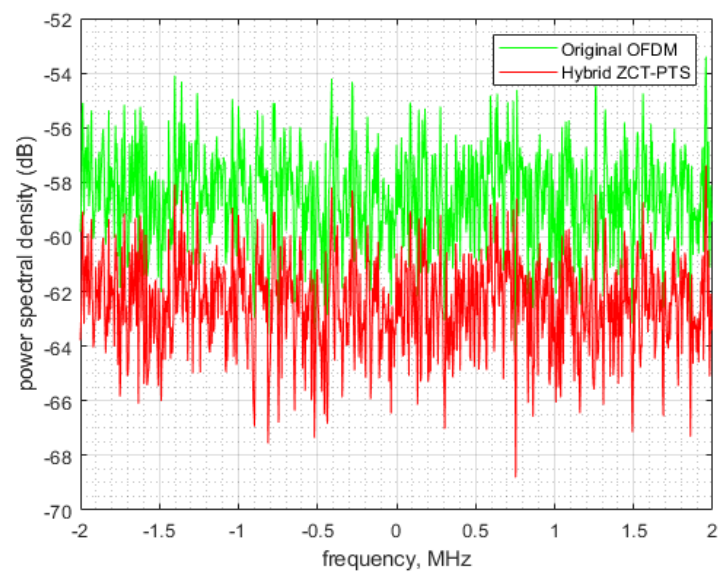

Fig.13. PSD of original OFDM and hybrid ZCT-PTS.

Table II is the comparative analysis of the developed hybrid ZCT-PTS and other PAPR techniques. It is observed that at clip rate of $10^{-1}$ when $(N=64), L=4$ (Oversampling factor) and Pseudorandom partitioning scheme (PR-PTS), the original OFDM PAPR is $10.84 \mathrm{~dB}$. This value is very high for the OFDM signal as it causes saturation in the Power Amplifier. If the PTS scheme is used separately to reduce the high PAPR in the OFDM signal, it is observed that a large reduction was achieved when the number of $V=8$ at $4.4 \mathrm{~dB}$. Increasing the value of $V$, the computational efficiency of the PTS scheme reduces. As a result, increasing the value of $V$ is not advisable for system design. The problem of computational complexity in PTS due to a large number of subblocks is minimized by using minimum subblocks in PTS scheme. This is fully achieved by combining the Zadoff-Chu precoding technique with PTS with a small value of $V(V=4)$. The hybrid technique gives a large reduction of about $4.2 \mathrm{~dB}$. The result of hybrid technique when compared to other reduction technique, shows that it has better PAPR reduction than others. Therefore, the problem of PTS with high computational complexity is reduced by employing the ZCT precoding technique with PTS having a small number of $V$.

TABLE II: COMPARISON OF PAPR TECHNIQUES WITH DIFFERENT SUBBLOCKS

\begin{tabular}{cccc}
\hline $\begin{array}{c}\text { Parameter } \\
\text { CCDF }\end{array}$ & $\begin{array}{c}\text { Different } \\
\text { OFDM Technique }\end{array}$ & $\begin{array}{c}\text { Number of } \\
\text { subblocks }\end{array}$ & $\begin{array}{c}\text { PAPR } \\
(\mathrm{dB})\end{array}$ \\
\hline $10^{-1}$ & Original OFDM & - & 10.84 \\
$10^{-1}$ & PTS & 2 & 7.90 \\
$10^{-1}$ & PTS & 4 & 5.82 \\
$10^{-1}$ & PTS & 8 & 4.4 \\
$10^{-1}$ & ZCT & - & 4.8 \\
$10^{-1}$ & Hybrid ZCT-PTS & 4 & 4.2 \\
\hline
\end{tabular}

\section{CONCLUSION}

OFDM, a major type of MCM has been adopted by both wired and wireless communications to provide a high data rate. Though, the major problem of the OFDM signal that can limit its implementation in wireless communication is its high PAPR. In this paper, a hybrid of Zadoff-Chu precoding (ZCT) and Partial Transmit Sequence (PTS) developed to reduce PAPR in the OFDM system is presented. The foremost idea of the hybrid technique is to multiply the input data streams with Zadoff-Chu precoding matrix, the precoded data are partitioned into subblocks before applying to the PTS technique. The Phase factors were determined by a suboptimal combination algorithm to reduce the search for phase complexity. Extensive simulation in MATLAB is performed to evaluate the developed hybrid ZCT-PTS and the simulation result shows that the hybrid technique has a low PAPR as compared to PTS and Zadoff Precoding method. In terms of BER and PSD, the hybrid ZCT-PTS method has a low BER and PSD when compared to the original OFDM. The developed hybrid technique is therefore recommended for mobile communication systems since these systems required a low computational complexity and less PAPR.

\section{REFERENCES}

[1] F. Sandoval, G. Poitau and F. Gagnon, "Hybrid peak-to-averagepower ratio reduction techniques: Review and performance comparison," IEEE Access, vol. 5, no. 4, pp. 27145-27161, 2017.

[2] A. M. Rateb and M. Labana, "An optimal low complexity PAPR reduction technique for next generation OFDM systems," IEEE Access, vol. 7, no. 2, pp. 16406-16420, 2019.

[3] M. A. Oyegoke and Y. O. Olasoji, "Peak to Average Power Ratio Reduction Techniques in Orthogonal Frequency Division Multiplexing Principle: A Comprehensive Review," Journal of Multidisciplinary Engineering Science and Technology (JMEST), vol. 8, no. 6, pp. 14091-14106, 2021.

[4] Y. A. Jawhar, L. Audah, M. A. Taher, K. N. Ramli, N. S. Shah, M. Musa and M. S. Ahmad, "A Review of Partial Transmit Sequence for PAPR Reduction in the OFDM Systems," in In IEEE Access, Parit Raja, Malaysia, 2019.

[5] M. Rateb, S. K. Syed Yusof and N. Fisal, "Adaptive data distribution technique for enhancing MB-OFDM UWB link performance in detect and avoid environments," International Journal of Ultra Wideband Communications and Systems, vol. 2, no. 1, pp. 1-13, 2011.

[6] A. V. Manjula and K. N. Muralidhara, "Hybrid Zadoff-Chu and multilateral piecewise exponential companding transform-based PAPR reduction technique in OFDM systems," International Journal of Communication Systems, vol. 33, no. 2, pp. 1-13, 2019.

[7] A. H. Fadel, H. H. Razzaq and S. A. Mostafa, "A low complexity partial transmit sequence approach based on hybrid segmentation scheme," Bulletin of Electrical Engineering and Informatics, vol. 9, no. 6, pp. 2371- 2379, 2020.

[8] C. Sanchis-Borrás, M. T. Martinez-Ingles, J. M. Molina-Garcia-Pardo, J. P. García and J. V. Rodriguez, "Experimental Study of MIMOOFDM Transmissions at $94 \mathrm{GHz}$ in Indoor Environments," in In IEEE Access, Murcia,Spain, 2017.

[9] B. Farhang-Boroujeny and H. Moradi, "OFDM inspired waveforms for 5G," IEEE Communications Surveys \& Tutorials, vol. 18, no. 4, pp. 2474-2492, 2016.

[10] P. Guan, D. Wu, T. Tian, J. Zhou, X. Zhang, L. Gu and Y. Kishiyama, "5G field trials: OFDM-based waveforms and mixed numerologies," IEEE Journal on Selected Areas in Communications, vol. 35, no. 6, pp. 1234-1243, 2017.

[11] C. Y. Hsu and H. C. Liao, "Generalised precoding method for PAPR reduction with low complexity in OFDM systems," IET Communications, vol. 12, no. 7, pp. 796-808, 2018.

[12] K. Anoh, C. Tanriover and B. Adebisi, "On the optimization of iterative clipping and filtering for PAPR reduction in OFDM systems," in In IEEE Access, Manchester, U.K, 2017.

[13] P. Lavanya, P. Satyanarayana and M. Mohatram, "Hybrid Technique based PAPR Reduction in OFDM," International Journal of 
Management, Technology And Engineering, vol. 9, no. 6, pp. 19601966, 2020.

[14] S. H. Han and J. H. Lee, "An overview of peak-to-average power ratio reduction techniques for multicarrier transmission," IEEE wireless communications, vol. 12, no. 2, pp. 56-65, 2005.

[15] T. Jiang and Y. Wu, "An overview: Peak-to-average power ratio reduction techniques for OFDM signals," IEEE Transactions on Broadcasting, vol. 54, no. 2, pp. 257-258, 2008.

[16] B. M. Popovic, Spreading sequences for multi-carrier CDMA systems, 1997.

[17] S. H. Muller, R. W. Bauml, R. F. Fischer and J. B. Huber, "OFDM with reduced peak-to-average power ratio by multiple signal representation," in proceeding of International conference of Ann Telecommunication, Ntirberg, Germany, 1997. 